\title{
Metodologia para avaliação de desempenho de produtores de leite fundamentada na lógica Fuzzy
}

\author{
Methodology for performance evaluation of dairy farmers based on fuzzy logic
}

\author{
Fabio Mamoré Conde ${ }^{1}$, Tomas Daniel Rodriguez², Mariluce Paes-de-Souza ${ }^{3}$, \\ Theophilo Alves Souza Filho ${ }^{4}$
}

\author{
${ }^{1}$ Instituto Federal de Educação de Rondônia - Campus de Vilhena - Universidade Federal de \\ Rondônia- Rondônia ,Brasil \\ ${ }^{234}$ Departamento de Administração. Mestrado em Administração Universidade Federal de Rondônia- \\ Rondônia, Brasil
}

\begin{abstract}
Resumo
O artigo apresenta o resultado de uma pesquisa sobre a avaliação do desempenho de produtores de leite no estado de Rondônia, na qual foi testada a possibilidade do uso de uma metodologia de avaliação baseada na lógica fuzzy, como uma alternativa aos métodos tradicionais de avaliação. A pesquisa, de caráter exploratório, foi dividida em duas etapas: a primeira trata o domínio conceitual da proposição de metodologia, com conceitos sistematizados a partir da revisão da literatura; a segunda explicita o domínio operacional por meio de simulações. As atividades do domínio operacional tiveram como base os dados de 485 produtores de leite de 23 municípios de Rondônia, do ano produtivo de 2008 e coletados em 2009 pelo Centro de Estudos Interdisciplinar em Desenvolvimento Sustentável da Amazônia. Ao final da investigação, demonstra-se a viabilidade da proposta de metodologia fundamentada na lógica fuzzy, ao indicar o potencial de sucesso como ferramenta de auxílio na avaliação de desempenho dos produtores de leite. A metodologia proposta permite explicar melhor, fundamentalmente, aqueles casos que ficam nas fronteiras das classes de classificação dos métodos tradicionais da avaliação do desempenho.
\end{abstract}

Palavras-chave: avaliação de desempenho, lógica fuzzy, agronegócio

\begin{abstract}
The article presents the results of a research on the performance evaluation of dairy farmers in the state of rondônia, in which we tested the possibility of using an evaluation methodology based on fuzzy logic, as an alternative to traditional evaluation methods. this article demonstrates the results of a research that presents the fuzzy logic applied in the performance evaluation of dairy farmers; such as main objective and basis in the development of a methodology for evaluating performance. the research, exploratory study, was divided into two stages: the first deals with the conceptual domain of the proposition methodology, from the systematic literature review concepts; and second clarifies the operational domain through simulations. the activities of the operating domain took as their basis data from 485 dairy farmers from 23 municipalities in rondônia, productive 2008 and collected in 2009 by the centro de estudos interdisciplinar em desenvolvimento sustentável da amazônia. at the end of the investigation demonstrates the viability of the proposed methodology based on fuzzy logic, indicate the potential for success as a tool to aid in evaluating the performance of dairy farmers. the proposed methodology allows us to explain better, fundamentally, those cases that are on the borders of the classes of the classification of traditional methods of performance evaluation
\end{abstract}

Keywords: performance evaluation, fuzzy logic, agribusiness 


\section{Introdução}

Caracterizada como uma das mais tradicionais estruturas produtivas do País, segundo circular técnica publicada pela Embrapa Gado de Leite com autoria de Carvalho (2010), a indústria de alimentos contribuiu em 2009 com quase $10 \%$ do Produto Interno Bruto (PIB) do Brasil. O setor de laticínios se destaca entre os quatro principais: Derivados de Carne; Beneficiamento de Café, Chá e Cereais; Açúcares e Laticínios.

Pedra et al. (2008) apontam uma evolução da produção brasileira de leite no período de 1997 a 2006. Nesse período houve um crescimento de aproximadamente $36 \%$ - com elevação da produção de 18,6 bilhões de litros em 1997 para 25,4 bilhões de litros em 2006. Ao mesmo tempo, o Valor Bruto da Produção Agropecuária do setor passou de $R$ \$ 6 bilhões para $R \$ 16$ bilhões.

Diferentemente do aumento de produção nacional, a participação relativa de Rondônia no Valor Bruto da Produção agropecuária diminuiu de $52,8 \%$ para $43,5 \%$. O Estado possui também participação nacional minoritária, sendo responsável por $4,3 \%$ da produção total do Brasil (FGV, 2010).

Porém, internamente, Rondônia obteve aumento de $400 \%$ na produção de leite no período compreendido entre 1990 e 2007, segundo a Embrapa Gado de Leite (EGL, 2011). Mesmo com expressivo aumento interno da produção, em uma comparação com outros estados brasileiros, a produtividade de Rondônia é 10,8 vezes menor que Minas Gerais que apresenta 27 litros/vaca por dia e é líder do ranking nacional.

Outra característica relacionada à produção de leite e que ocorre em Rondônia é o baixo nível de escolaridade dos produtores. Entre os sócios fundadores das micro e pequenas empresas da cadeia produtiva do leite $35,1 \%$ possuem ensino fundamental incompleto e $28,6 \%$ ensino médio completo (PAES SOUZA et al., 2006). Com base na mesma pesquisa, a contratação de empregados qualificados é uma das principais dificuldades para $37,7 \%$ dos respondentes. Esses elementos demonstram a necessidade de estudos que investiguem ferramentas de gestão que tenham como pressuposto atender a particularidade deste público.

Porém, apesar da baixa produtividade de Rondônia, os dados evidenciam a importância e o aumento do setor lácteo na região. Nessa perspectiva, considerase que métodos de avaliação de desempenho podem ser caracterizados como artefatos centrais no processo de tomada de decisão. A pesquisa tem o objetivo de desenvolver uma metodologia de avaliação de desempenho dos produtores de leite fundamentada na lógica fuzzy, procedendo-se a: elaboração do quadro teórico; definição dos fatores e dimensões utilizando a análise fatorial; cálculos da eficiência dos produtores com o método análise envoltória de dados; identificação de clusters de eficiência desses produtores; validação da metodologia a partir de testes estatísticos efetuados com os resultados obtidos na metodologia proposta com a análise envoltória de dados.

Para estruturação deste artigo, optou-se por organizar em cinco tópicos, a contar da parte introdutória; os conceitos preliminares acerca dos artefatos que compõem a proposta; seguidos do detalhamento metodológico que orienta a pesquisa; os resultados das simulações de desempenho e respectivas discussões; as conclusões e, por fim, as referências.

\section{Conceitos Preliminares}

A medição de desempenho pode ser entendida, de maneira geral, como a técnica usada para quantificar a eficiência e a eficácia das atividades de negócio Neely et al. (1995). A eficácia avalia o resultado do cumprimento das metas definidas e a eficiência trata da relação entre a utilização econômica dos recursos e os resultados alcançados, quando comparados com outras unidades com as mesmas atividades.

Observa-se que na literatura existem diversas perspectivas para os modelos de sistemas de medição e avaliação de desempenho. Bogan e English (1996) relacionam o planejamento estratégico com a medição de desempenho efetuado pelo benchmarking estratégico que serve como uma bússola de navegação muito útil.

Sink e Tuttle (1993) extrapolam a simples formulação da estratégia e a relacionam com a sua execução. Os autores destacam que a estratégia de negócio bem compreendida pode impulsionar a constante melhoria da performance a partir da utilização de sistemas de medição bem projetados e desenvolvidos. Os autores defendem também que a medição pode ser um impulso muito eficaz para a melhoria da performance.

Fontes e Mello (2004) destacam que duas características são importantes na avaliação de desempenho: 1) indicar que o produtor têm um bom desempenho naquilo que é melhor; e 2) não pode ter um mau desempenho no atributo em que for pior.

Uma técnica utilizada para medição de desempenho é a Análise Envoltória de Dados (DEA, Data Envelopment Analysis). Segundo Vilela et al. (2007), o marco inicial sobre DEA pode ser encontrado em The Measurement of Productive Efficiency, de Farrell em 1957, quando Farrell propôs um modelo empírico para cálculo da eficiência relativa. O modelo considerou um único insumo e um único produto. Ainda segundo o autor, Charnes (1978) iniciou o estudo com múltiplos insumos (inputs) e múltiplos produtos (outputs), o que denominou então como Análise Envoltória de Dados (DEA).

O DEA tem como objetivo comparar certo número de Unidades Tomadoras de Decisão (DMUs, Decision Making Units) que realizam tarefas similares e se diferenciam nas quantidades de inputs (entradas, recursos, insumos ou atores de produção) que consomem e de outputs (saídas ou produtos) que produzem (Gomes e 
Mangabeira, 2004).

A metodologia DEA utiliza programação linear para estimar uma fronteira eficiente. Existem dois modelos DEA clássicos: a) o modelo que trabalha com retornos constantes de escala (CCR, Charnes, Cooper e Rhodes) (CHARNES, 1978) e assume proporcionalidade entre inputs e outputs; b) o modelo que considera retornos variáveis de escala (BBC, Banker, Chanes e Cooper) (GOMES; MANGABEIRA, 2004); isto é, substitui o axioma da proporcionalidade pelo axioma da convexidade.

A formulação matemática para o modelo BCC é apresentada em (1). Nesta formulação, ho é a eficiência da DMUo em análise; xik representa o input i da DMUk, yjk representa o output j da DMUk; vi é o peso atribuído ao input $\mathrm{i}$, uj é o peso atribuído ao output j; $\mathrm{u}^{*}$ é um fator de escala (PEÑA, 2008).

$$
\begin{aligned}
& \max h_{o}=\sum_{j=1}^{m} u_{j} y_{j o}+u^{*} \\
& \text { sujeito a } \\
& \sum_{i=1}^{n} v_{i} x_{i o}=1 \\
& \sum_{j=1}^{m} u_{j} y_{j k}-\sum_{i=1}^{n} v_{i} x_{i k} \leq 0, \quad k=1, \ldots, s \\
& u_{j}, v_{i} \geq 0 \quad \forall x, y \\
& u^{*} \in \Re
\end{aligned}
$$

Porém, para identificar quais variáveis são relevantes na pesquisa, utiliza-se a Análise Fatorial (AF). A AF pode ser vista como um termo que tem como finalidade básica a redução e sumarização de dados. Ramos (2010) esclarece que a análise fatorial não denomina uma única técnica estatística, mas diversas técnicas que visam facilitar a interpretação dos dados. A estrutura de relações da $\mathrm{AF}$, segundo o mesmo autor, utiliza duas variações:

1. Análise de fatores - visa determinar um número mínimo de fatores para explicar o máximo de variação dos dados, tendo conhecimento prévio de que as variâncias específicas e do erro são pequenas com relação à variância total.

2. Análise de componentes principais - visa identificar dimensões latentes no conjunto de variáveis, tendo pouco conhecimento sobre as variâncias específicas e do erro, desejando eliminá-las.

Moori e Zilber (2003) destacam que para aplicar a AF inicialmente é necessário verificar algumas condições sobre o conjunto de dados: a) a distribuição de frequência das variáveis; b) à consistência ou confiabilidade do fator gerado em torno das variáveis; c) comunalidade, que são quantidades das correlações de cada variável explicada pelos fatores.

De acordo com Rezende et al. (2007) referenciando a Sharma (1996), no modelo de análise fatorial, apresentado em (2), cada uma das n variáveis representa uma combinação linear de $\mathrm{m}$ fatores comuns e de um fator específico. Para o i-ésimo indicador tem-se:

$$
x_{i j}=\sum_{p=1}^{m} a_{i p} f_{p j}+u_{i} y_{i j}
$$

onde:

fpj é o valor do p-ésimo fator comum para a j-ésima observação;

aip (com $\mathrm{p}=1, \ldots, \mathrm{m})$ é o coeficiente dos fatores comuns;

ui é coeficiente dos fatores específicos;

yij representa o j-ésimo valor do i-ésimo fator específico, ou seja, é o valor único que representa a parte não explicada pelos fatores comuns.

Para auxiliar na interpretação dos fatores obtidos com a AF pode ser efetuada sua rotação. Após a rotação, para cada novo fator é apresentada uma correlação relativamente forte com uma ou mais variáveis e outra relativamente fraca com as demais variáveis.

Um dos problemas no tratamento de dados é a sua imprecisão. Zadeh (1965) observa que é frequente que um objeto do mundo real não seja precisamente definido como membro de apenas uma classe, pois possui características ambíguas. Da mesma forma, Campos Filho (2004) elucida que a lógica fuzzy tem por objetivo fornecer um instrumento que contemple os aspectos imprecisos no raciocínio lógico dos seres humanos em situações ambíguas.

Matematicamente, um conjunto fuzzy $C$ é definido por uma função de pertinência com intervalo entre 0 e 1, representado por $\mu \mathrm{C}:[0-1]$. Essa função associa a cada elemento y de $\mu \mathrm{C}:[0-1]$ um grau de pertinência, representado por $\mu \mathrm{C}(\mathrm{y})$ (SANDRI e CORREA, 1999):

- $\mathrm{SE} \mu \mathrm{C}(\mathrm{y})=0$, y é completamente incompatível com conjunto fuzzy;

- $\quad \mathrm{SE} \mu \mathrm{C}(\mathrm{y})=1$, y é completamente compatível com conjunto fuzzy; e

- $\mathrm{SE} \mu \mathrm{C}(\mathrm{y})>0$ e $\mu \mathrm{C}(\mathrm{y})<1$ indica que y é parcialmente compatível com conjunto fuzzy, com grau $\mu \mathrm{C}(\mathrm{y})$.

Essas características implicam em considerar um conjunto contínuo de valores de pertinência, formando um agrupamento onde a transição de não pertinência para pertinência é gradual. O grau de pertinência é definido por funções, representadas por parâmetros específicos da função. Um dos formatos possíveis é a função gaussiana, apresentada por (3).

$$
\mu_{\mathrm{A}}(x)=e^{-k(x-m)^{2}} ;(k>1)
$$

e graficamente mostrada na Figura 01. 


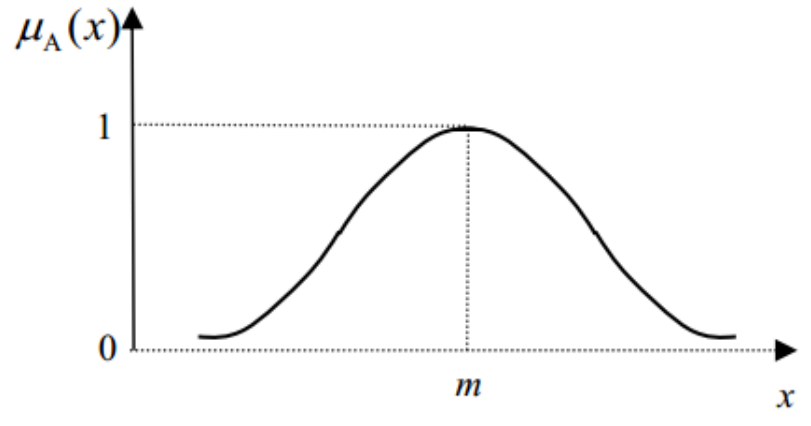

Figura 01 - Função Gaussiana.

A lógica fuzzy utiliza elementos de linguagem natural ao invés de valores quantitativos. Uma variável linguística tem por característica assumir valores dentro de um conjunto linguísticos, ou seja, palavras ou frases como, por exemplo: "baixa", "média", "alta".

As variáveis linguísticas formam as regras declaradas na forma

SE $<$ condição $>$ ENTÃ $\mathrm{C}$ resultado $>$

e descrevem a ação a ser feita em resposta as várias de entradas. As regras formam uma base de conhecimento que caracteriza a estratégia de inferência do universo desejado.

De forma geral, Shaw e Simões (2007) apresentam um modelo conceitual de como operar o Controlador de Lógica Fuzzy. Os autores destacam que o mecanismo primordial desse modelo consiste em introduzir ao universo nebuloso as variáveis discretas (processo de fuzzificação), representadas por escalas numéricas, processá-las com base em regras estabelecidas com o auxílio de informações de especialistas. Posteriormente, estas são resgatadas no formato de saídas discretas (processo de defuzzificação), ou seja, em números representativos para tomada de decisão.

Rodrigues e Santos (2004) relatam que a área da administração possui poucos estudos que utilizam a lógica fuzzy, dentre os existentes podem-se citar os seguintes estudos: priorizar objetivos e ações estratégicas organizacionais (Vanti et al., 2007); analisar projetos turísticos (Medeiros, et al., 2007); investigar os indicadores de fertilidade do solo cultivado com café (Assis Silva e Souza Lima, 2009).

No agronegócio, a lógica fuzzy vem sendo aplicada com viabilidade positiva nos seguintes estudos: interpretar o índice de massa corporal de qualquer tipo de espécie bovina e em qualquer região do País (GABRIEL FILHO, 2011); desenvolver uma metodologia de classificação automática utilizando indicadores de vegetação de imagens (ANTUNES e ZULLO JÚNIOR, 2007); verificar a necessidade de aeração de grãos de milho de forma racional em silos verticais (DIA et al., 2005); estimar um padrão ideal de conforto ambiental para creche de suínos (QUEIRÓS e NÄÄS, 2005); propor e avaliar uma metodologia para a classificação da compactação do solo (ARAÚJO et al., 2005).

\section{Metodologia}

O objetivo que norteou esta pesquisa de caráter exploratório foi o desenvolvimento e validação de uma metodologia de avaliação de desempenho fundamentada na lógica fuzzy. Destina-se a ser aplicada como uma ferramenta para a análise dos produtores de leite de Rondônia.

A amostra de dados é composta por produtores de leite do estado de Rondônia, com produção no ano de 2008 e coletados em 2009 pelo Centro de Estudos Interdisciplinar em Desenvolvimento Sustentável da Amazônia (CEDSA). São dados de 23 municípios de Rondônia com um total de 485 entrevistados e que serviram para a validação deste artefato através de simulações.

O uso dessa amostra é justificado pelo fato de se verificar um volume de investimentos na atividade leiteira, por parte dos governos Federal e Estadual (Rodrigues, 2010). Por serem informações levantadas por um centro de pesquisa e destinados ao diagnóstico de investimentos, isso lhes confere confiabilidade.

Na presente pesquisa a definição das variáveis foi originada no estudo de Rodrigues (2010): Volume de produção anual (VPA), Renda bruta (R\$/ano), Produtividade das vacas (PV) (litros/hectare/ano), Produtividade da terra (litros/hectare/ano), Produtividade do capital investido (litros/R\$), Produtividade da mão-de-obra (litros/R\$), Produtividade do custo operacional total (litros/ $\mathrm{R} \$)$, Participação da renda leite (\%), Custo operacional total (R\$/ano) e Custo unitário de produção (R\$/ano).

Os indicadores utilizados estão em escalas diferentes, portanto, com o objetivo de evitar a influência das unidades de medidas, as variáveis foram previamente normalizadas. Foram aplicadas as técnicas de análise fatorial, análise envoltória de dados, análise de conglomerados e lógica fuzzy.

Primeiramente, na aplicação da análise fatorial, utilizou-se o método de análise de componentes principais que tem como vantagem a identificação do quanto cada variável está relacionada a cada fator (ARTE, 1998). Para melhor interpretação dos fatores gerados foi executado o método Varimax. Posteriormente, foi efetuada a analise do valor do Kaiser-Meyer-Olkin (Measure of Sampling Adequacy - MSA) de cada indicador. Este valor indica o grau de explicação dos dados a partir dos fatores encontrados. Segundo Bezerra e Corrar (2006), valores abaixo de 0.50 são considerados muito pequenos para análise e indicam variáveis que podem ser retiradas da análise.

O próximo passo foi executar o cálculo da análise envoltória de dados e para isso definiu-se duas matrizes 
de dados: 1) formada por $\mathrm{k}$ insumos, utilizados por $\mathrm{n}$ produtores; e 2) formada por $\mathrm{m}$ produtos, produzido por $\mathrm{n}$ produtores. O cálculo da eficiência foi efetuado com base no resultado da Análise Fatorial. As características do DEA presentes nesse estudo são: orientação para produto; indicador de eficiência do Modelo BCC (pois não assume proporcionalidade entre insumo e produto). Com a definição do cenário de análise, foi calculada a eficiência dos produtores de leite da amostra. Dos quatro índices possíveis foi considerado alvo a Eficiência Composta Normalizada por ser a medida mais robusta de eficiência do modelo DEA (Steffanello, Macedo e Alyrio, 2009). Este índice é definido como a relação entre o valor da eficiência composta de cada unidade e o valor da eficiência composta da unidade mais eficiente.

Na etapa seguinte calculou-se a Análise de Conglomerados. Foram definidos sete agrupamentos de acordo com a escala de Matarazzo (1998 apud BORINELLI, 1989): Ótimo, Bom, Satisfatório, Razoável, Fraco, Deficiente e Péssimo. Utilizou-se o método não hierárquico K-Médias que possui como referência de distância entre as classes o valor de centroide das observações (Corrar, Paulo, Diasfilho, 2001). O objetivo da aplicação desta técnica é a identificação de segmentos homogêneos de produtores e, a partir desse resultado, formar limiares entre as classes de eficiência com extremos de ineficientes (0) e eficientes (1). A análise de conglomerado foi executado no resultado da análise fatorial, da análise envoltória de dados e da lógica fuzzy.

Foram realizadas duas simulações com a finalidade de validar operacionalmente a abordagem de avaliação de desempenho proposta nesta pesquisa. A finalidade de realizar duas simulações é identificar se: 1) estratégias diferentes de construção de máquinas de inferência, a partir dos mesmos indicadores, pode ser adotada; e 2) reduzir o quantitativo de regras de inferência necessárias. Para a lógica fuzzy, nas duas simulações foram utilizados o Método de Mamdani, na etapa de inferência das regras; o Método do centro de gravidade, na etapa de defuzzificação; e a função de pertinência a Gaussiana.

Operacionalmente os dados de cada produtor foram tabulados no software Microsoft Excel, de forma a possibilitar seleção e análise das variáveis. Neste software também foram calculados os testes estatísticos (1) ANOVA: Fator único e (2) Teste - Z: Duas amostras para médias, $\operatorname{com} P(Z<=z)$ uni-caudal de diferença entre as médias. Os dados para cálculo possuem distribuição normal, variância semelhante e são independentes. Ambos os teste com nível de significância igual 0,05 com o intuito de detectar se existem diferenças significativas entre as médias e variâncias das variáveis dos produtores estudados segundo as técnicas aplicadas. O software estatístico SPSS $\AA$ - Statistical Package for the Social Sciences versão 17.0 foi empregado para o cálculo da Análise Fatorial e da Análise de Conglomerados. O software SIADv3 (Sistema Integrado de Apoio à Decisão) foi utilizado para calcular os resultados da eficiência da Análise Envoltória de Dados. A construção da estrutura da lógica fuzzy foi efetuada no software MATLAB ${ }^{\circledR}$ R2012a Student Version. O MATLAB ${ }^{\circledR}$ dispõe de vários toolboxes e dentre os existentes encontra-se o Fuzzy Logical Toolbox que disponibiliza funções destinadas ao uso da teoria de conjuntos fuzzy.

\section{Resultados e discussões}

Seguindo a metodologia especificada e com a utilização de softwares estatísticos, chegou-se às seguintes proposições e simulação.

\subsection{Proposta para Avaliação do Desempenho dos Produtores de Leite Fundamentado na Lógica Fuzzy}

Apesar da diversidade de metodologias de avaliação de desempenho, é possível identificar uma carência de técnicas aptas a avaliar o desempenho de pequenos produtores de leite e que possibilitem minimizar o impacto da imprecisão dos dados de entrada da avaliação e fazer a gradual classificação entre os resultados.

Frente a esta necessidade é apresentada uma metodologia especializada de avaliação de desempenho, fundamentada na lógica fuzzy. Para tanto, esta metodologia utiliza também a análise fatorial, análise de conglomerados e a análise envoltória de dados para preparação e controle do modelo.

A partir da sistematização de uma metodologia geral de medição de desempenho elaborada por de Sink e Tuttle (1993), definiu-se o fluxo de processos da metodologia de avaliação de desempenho proposto. Como resultado da pesquisa, apresenta-se na Figura 02 o diagrama relativo aos módulos da metodologia proposta e que representa o enfoque conceitual desta investigação.

A organização apresentada na Figura 02 sugere uma estrutura modularizada para a metodologia proposta. Assim, quatro módulos compõem esse modelo e possuem questões como ponto de partida, sendo estas:

Módulo 1 - O que Medir? Para isso, é utilizado a técnica estatística multivariada de Análise Fatorial.

Módulo 2 - Qual a referência da avaliação? O método sugerido para responder é a Análise de Conglomerados e a Análise Envoltória de Dados.

Módulo 3 - Qual a método principal? A avaliação proposta é fundamentada na Lógica fuzzy.

Módulo 4 - Para finalizar, emerge a interrogação: Está correto? Para isso, serão utilizadas técnicas estatísticas para testar e validar as hipóteses de trabalho: Teste Z e Cálculo da Anova.

No domínio operacional da metodologia, os módulos são executados em etapas: 1) identificam-se (na base de produtores de leite) com o método de análise fatorial 


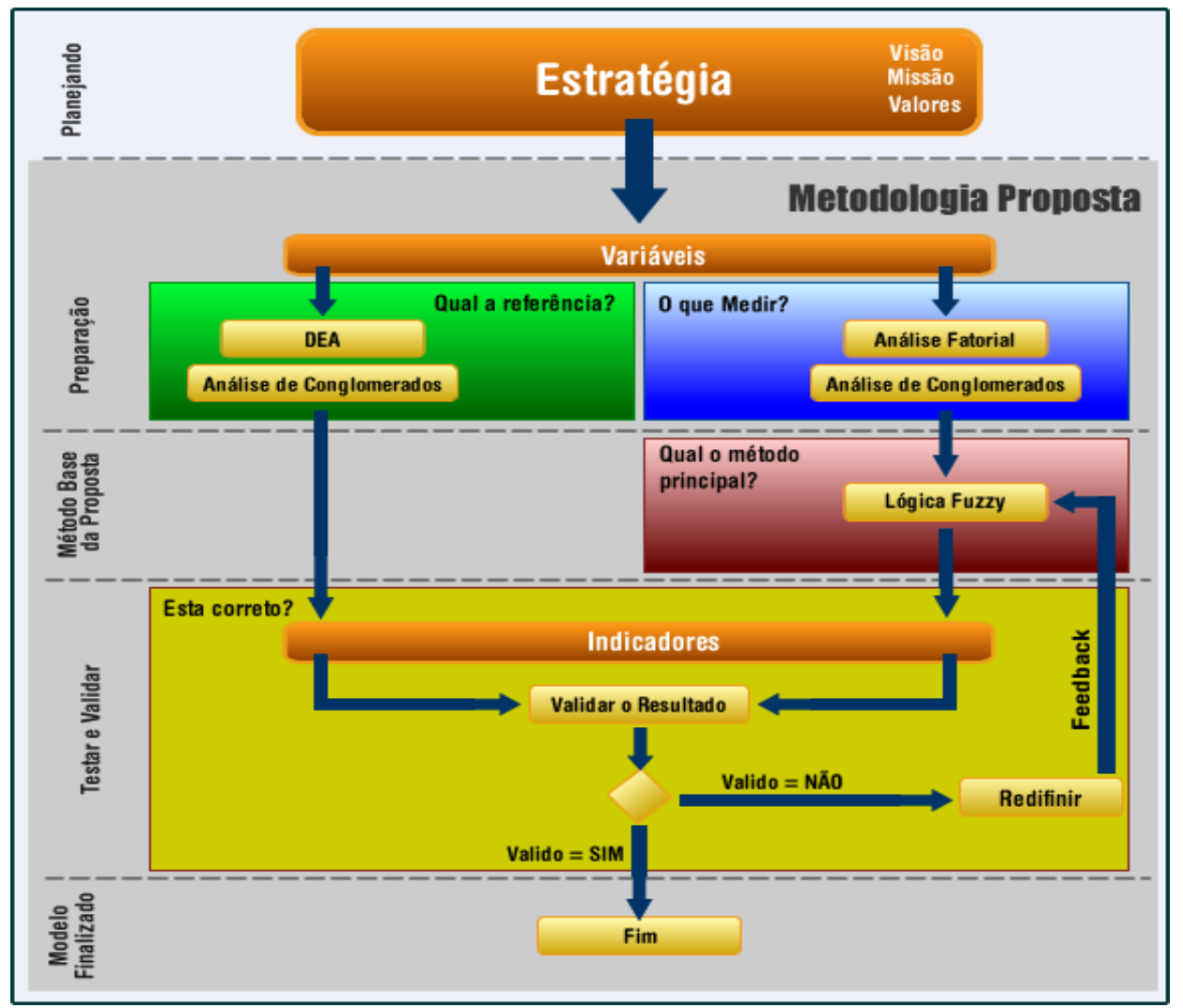

Figura 02: Visão global de medição segundo a metodologia proposta.

(AF) quais variáveis são mais significativas; 2) calculase a eficiência dos produtores de leite com o método de análise envoltório de dados (DEA); 3) são definidas as classes de eficiência (sobre as variáveis calculadas pela AF e DEA) com o método de análise de conglomerados; 4) elaboração do modelo de avaliação de desempenho fundamentado na lógica fuzzy; 5) validação pelos testes Anova e Teste-Z.

Após essas etapas, o modelo de avaliação criado é revisado e novas regras de inferência são incorporadas e/ou eliminadas, formando um processo cíclico entre as etapas 4 e 5 . O ciclo de construção do modelo de avaliação fuzzy finaliza quando os testes estatísticos indicam que não há diferença significativa entre a avaliação de desempenho desenvolvida e a análise envoltória de dados.

\subsection{Simulação da Metodologia Proposta}

Como definido na metodologia de pesquisa, foram efetuadas duas simulações na qual definiram-se estratégias diferentes de organização dos indicadores de entrada. Os Módulos 1 e 2 são os pilares na metodologia e serviram de base para as duas simulações. Neste sentido, o Módulo 3 - que aplica a lógica fuzzy - e o Módulo 4 - que valida o modelo de avaliação - são específicos em cada simulação.
Em resumo, a estratégia de organização dos indicadores é: simulação 1 - definida de acordo com os fatores/dimensões encontrados no Módulo 1; simulação 2 - definida subjetivamente.

\subsubsection{Módulo 1: Medição através da Análise Fatorial}

Como definido na metodologia, neste módulo foi calculada a AF nas dez variáveis normalizadas e previamente foram efetuadas algumas validações: 1) Determinante de 0,376 (calculado com a Matriz de Correlação) indica que não há variável dependente, possibilitando, com isso, o cálculo da $\mathrm{AF}$ e a inversão da matriz; 2) KMO (Kaiser-Meyer-Olkin) de 0,605 demonstra que é possível a utilização da AF; 3) Significância igual a 0,000 indica que os dados são apropriados para o cálculo.

Como resultado da AF obteve-se cinco variáveis distribuídas em quatro fatores. Observa-se que as explicações das variáveis a partir dos fatores calculados (Comunalidades) estão acima de 0.857 . A variação total da AF demostra que os quatro fatores identificados explicam $94 \%$ da variação dos indicadores. Nota-se que essas quatro dimensões calculadas pela AF explicam quase a totalidade da variação dos dados.

O próximo exame foi referente à relação dos indicadores e dos fatores calculados e para isso utilizou-se 
Quadro 01: Resultado da Análise Fatorial

\begin{tabular}{|l|l|}
\hline \multicolumn{2}{|c|}{ Resultado da Análise Fatorial } \\
\hline \multicolumn{2}{|c|}{ Fator 1: Custo } \\
\hline O fator que sugere um maior controle dos custos é responsável por 34.22\% da variância explicada. \\
\hline Custo operacional total: & $\begin{array}{l}\text { Valor em reais do custo operacional total do ano; somatória do } \\
\text { custo operacional efetivo mais a depreciação de máquinas e } \\
\text { benfeitorias e a mão-de-obra familiar; }\end{array}$ \\
\hline Custo unitário de produção: & $\begin{array}{l}\text { O custo operacional por unidade produzida; relação entre custo } \\
\text { operacional total e volume de produção anual. }\end{array}$ \\
\hline \multicolumn{2}{|c|}{ Fator 2: Animal } \\
\hline O fator que sugere um maior controle dos animais é responsável por 20.12\% da variância explicada. \\
\hline Produtividade das vacas: & $\begin{array}{l}\text { Quantidade de produção anual de leite em litros por unidade de } \\
\text { vaca na propriedade; relação entre a produção leite anual e o } \\
\text { número de vacas (litros/vaca); }\end{array}$ \\
\hline \multicolumn{1}{|c|}{ Fator 3: Investimento } \\
\hline O fator que sugere um maior controle dos investimentos é responsável por 20.03\% da variância \\
explicada.
\end{tabular}

a matriz dos componentes após a rotação dos fatores (Rotated Component Matrix). A rotação permitiu uma classificação mais precisa dos indicadores em cada um dos fatores.

No Quadro 01 é apresentado o resultado da Análise Fatorial com a utilização do método de Análise de Componentes Principais.

\subsubsection{Módulo 2: Referência com base na Análise Envoltória de Dados}

Neste módulo foi calculada a eficiência dos produto- res de leite. O Quadro 02 mostra que a avaliação padrão identifica três unidades como eficientes, enquanto a eficiência composta normalizada (Composta*) identifica apenas uma DMU como eficiente. Esta característica indica maior seletividade de unidades eficientes e portanto selecionada como índice de referência na pesquisa.

Após o calculo da eficiência foi efetuada a classificação pelo método de análise de conglomerados. A análise de conglomerados permitiu classificar, além da eficiência do DEA, todas as variáveis em classes de eficiência. Com isso, identificou-se intervalos de valores para cada classe, com exemplo mostrado no Quadro 03.

Quadro 02: Exemplo de Avaliação DEA.

\begin{tabular}{|l|l|l|l|l|}
\hline $\begin{array}{l}\text { DMU } \\
\text { (Produtor de } \\
\text { Leite) }\end{array}$ & $\begin{array}{l}\text { Eficiência } \\
\text { Padrão }\end{array}$ & $\begin{array}{l}\text { Eficiência } \\
\text { Invertida }\end{array}$ & $\begin{array}{l}\text { Eficiência } \\
\text { Composta }\end{array}$ & $\begin{array}{l}\text { Eficiência } \\
\text { Composta* }\end{array}$ \\
\hline 122 & 1 & 0,022712 & 0,988644 & 1 \\
\hline 99 & 1 & 0,042149 & 0,978926 & 0,99017 \\
\hline 132 & 1 & 0,06 & 0,97 & 0,981142 \\
\hline
\end{tabular}


Quadro 03: Variável Quantitativa x Variável Linguística.

\begin{tabular}{|c|c|c|c|c|c|c|c|}
\hline $\begin{array}{l}\text { Classe / } \\
\text { Indicador }\end{array}$ & Ótimo & Bom & Satisfatório & Razoável & Fraco & Deficiente & Péssimo \\
\hline $\begin{array}{l}\text { Custo } \\
\text { Operacional } \\
\text { Total }\end{array}$ & $\begin{array}{l}\text { Variável <= } \\
9450\end{array}$ & $\begin{array}{l}9450< \\
\text { Variável } \\
<= \\
19500\end{array}$ & $\begin{array}{l}19500< \\
\text { Variável <= } \\
30390\end{array}$ & $\begin{array}{l}30390< \\
\text { Variável <= } \\
44500\end{array}$ & $\begin{array}{l}44500< \\
\text { Variável } \\
<= \\
58650\end{array}$ & $\begin{array}{l}58650< \\
\text { Variável <= } \\
79000\end{array}$ & $\begin{array}{l}\text { Variável } \\
>79000\end{array}$ \\
\hline $\begin{array}{l}\text { Custo } \\
\text { Unitário de } \\
\text { Produção }\end{array}$ & $\begin{array}{l}\text { Variável <= } \\
2,14881\end{array}$ & $\begin{array}{l}2,14881 \\
< \\
\text { Variável } \\
<= \\
4,35185\end{array}$ & $\begin{array}{l}4,351852< \\
\text { Variável <= } \\
5,865385\end{array}$ & $\begin{array}{l}\text { 5,865385 } \\
<\text { Variável } \\
<= \\
7,135833\end{array}$ & $\begin{array}{l}7,13583 \\
< \\
\text { Variável } \\
<= \\
7,51467\end{array}$ & $\begin{array}{l}7,514677 \\
<\text { Variável <= } \\
8,037037\end{array}$ & $\begin{array}{l}\text { Variável > } \\
8,0373 \\
7\end{array}$ \\
\hline
\end{tabular}

Quadro 04: Relação da variável quantitativa para variável linguística.

\begin{tabular}{|l|l|l|l|l|l|l|}
\hline DMU & $\begin{array}{l}\text { Custo } \\
\text { operacional } \\
\text { total }\end{array}$ & $\begin{array}{l}\text { Custo } \\
\text { unitário de } \\
\text { produção }\end{array}$ & $\begin{array}{l}\text { Produtividade } \\
\text { das vacas }\end{array}$ & $\begin{array}{l}\text { Produtividade } \\
\text { do capital }\end{array}$ & $\begin{array}{l}\text { Prod. do } \\
\text { custo } \\
\text { operacional }\end{array}$ & $\begin{array}{l}\text { Avaliação } \\
\text { DEA }\end{array}$ \\
\hline 122 & Ótimo & Ótimo & Ótimo & Satisfatório & Razoável & Ótimo \\
\hline 145 & Bom & Ótimo & Satisfatório & Satisfatório & Deficiente & Bom \\
\hline 67 & Bom & Ótimo & Bom & Razoável & Péssimo & Satisfatório \\
\hline 152 & Ótimo & Ótimo & Fraco & Deficiente & Razoável & Razoável \\
\hline 75 & Bom & Ótimo & Fraco & Deficiente & Péssimo & Fraco \\
\hline 114 & Satisfatório & Bom & Deficiente & Péssimo & Péssimo & Deficiente \\
\hline 35 & Deficiente & Bom & Deficiente & Péssimo & Péssimo & Péssimo \\
\hline
\end{tabular}

Com os intervalos calculados, foi identificado o vínculo entre o valor quantitativo da base de dados com as variáveis linguísticas da lógica fuzzy. Com base nos dados de produtores de leite, alguns exemplos da conversão da variável quantitativa para variável linguística são apresentados no Quadro 04.

\subsubsection{Módulo 3: Lógica Fuzzy como método princi-} pal

Como especificado na metodologia de avaliação de desempenho proposta, a partir deste módulo inicia-se o ciclo de construção e reconstrução da estrutura da lógica fuzzy: funções de pertinência e base de regras. Inicialmente é necessário a definição do universo do discurso de cada variável. Esta definição foi efetuada a partir da base de dados dos produtores, com resultado apresentado no Quadro 05.

Como definido, foram estruturadas duas simulações para validação da proposta. $\mathrm{O}$ detalhamento da organização dos indicadores é apresentado no Quadro 06 e mostra a interrelação dos indicadores em cada máquina de inferência.

No Quadro 07 é apresentado o quantitativo de regras definidas para cada máquina de inferência, formando com isso a base de conhecimento da lógica fuzzy - elaborada a partir dos dados dos produtores de leite. O total de regras é definido pela relação entre o quantitativo de variáveis de entrada e o número de variáveis linguísticas.

Com a construção das máquinas de inferência, o desempenho dos produtores de leite foi calculado utilizando-se a lógica fuzzy. No Quadro 08 são mostrados 
Quadro 05: Universo do Discurso.

\begin{tabular}{|l|l|}
\hline Indicador & Universo \\
\hline Custo operacional total & {$[150 . .100450]$} \\
\hline Custo unitário de produção & {$[0,0166 . .8,998611]$} \\
\hline Produtividade das vacas & {$[49,0909 . .7880]$} \\
\hline Produtividade do capital & {$[0,0568 \ldots 18]$} \\
\hline Prod. do custo operacional & {$[0,1111 \ldots 60]$} \\
\hline
\end{tabular}

Quadro 06: Organização dos Indicadores.

\begin{tabular}{|l|l|}
\hline Simulação 1 & Simulação 2 \\
\hline Primeira Máquina de Inferência \\
\hline $\begin{array}{l}\text { Parâmetros de entrada: os indicadores custo } \\
\text { operacional total e custo unitário de produção. } \\
\text { Resultado: Avaliação do Custo. }\end{array}$ & $\begin{array}{l}\text { Parâmetros de entrada: os indicadores custo } \\
\text { operacional total e custo unitário de produção. } \\
\text { Resultado: Avaliação do Custo. }\end{array}$ \\
\hline $\begin{array}{l}\text { Segunda Máquina de Inferência } \\
\text { Parâmetros de entrada: os indicadores avaliação do } \\
\text { custo, produtividade das vacas, produtividade do } \\
\text { capital investido e produtividade do custo } \\
\text { operacional total. }\end{array}$ total. \\
Resultado final: Avaliação do Desempenho. investido e produtividade do custo operacional \\
\hline Terceira Máquina de Inferência & Resultado: Avaliação do Dinheiro. \\
\hline
\end{tabular}

Quadro 07: Base de Regras.

\begin{tabular}{|c|c|}
\hline Simulação 1 & Simulação 2 \\
\hline $\begin{array}{l}\text { Para Avaliação do Custo: Com dois Indicadores e } \\
\text { sete variáveis linguísticas }\left(7^{2}\right) \text { foram definidas } 49 \\
\text { (quarenta e nove) regras de inferência; }\end{array}$ & $\begin{array}{l}\text { Para Avaliação do Custo e Avaliação do Dinheiro: Com } \\
\text { dois indicadores e sete variáveis linguísticas }\left(7^{2}\right) \text { foram } \\
\text { definidas } 49 \text { (quarenta e nove) regras de inferência para } \\
\text { cada avaliação; }\end{array}$ \\
\hline $\begin{array}{l}\text { Para Avaliação de Desempenho: Com quatro } \\
\text { indicadores e sete variáveis linguísticas }\left(7^{4}\right) \text { foram } \\
\text { definidas } 2401 \text { (duas mil quatrocentas e uma) } \\
\text { regras de inferência. }\end{array}$ & $\begin{array}{l}\text { Para Avaliação de Desempenho: Com três indicadores e } \\
\text { sete variáveis linguísticas }\left(7^{3}\right) \text { foram definidas } 343 \\
\text { (trezentas e quarenta e três) regras de inferência. }\end{array}$ \\
\hline
\end{tabular}


Quadro 08: Avaliação de Desempenho.

\begin{tabular}{|l|l|l|l|}
\hline DMU & DEA & $\begin{array}{l}\text { Simulação 1 } \\
\text { FUZZY Fatores }\end{array}$ & $\begin{array}{l}\text { Simulação 2 } \\
\text { FUZZY Subjetivo }\end{array}$ \\
\hline 122 & 1,000 & 0,812 & 0,967 \\
\hline 99 & 0,990 & 0,594 & 0,835 \\
\hline 132 & 0,981 & 0,451 & 0,793 \\
\hline 221 & 0,796 & 0,494 & 0,741 \\
\hline 145 & 0,715 & 0,627 & 0,645 \\
\hline
\end{tabular}

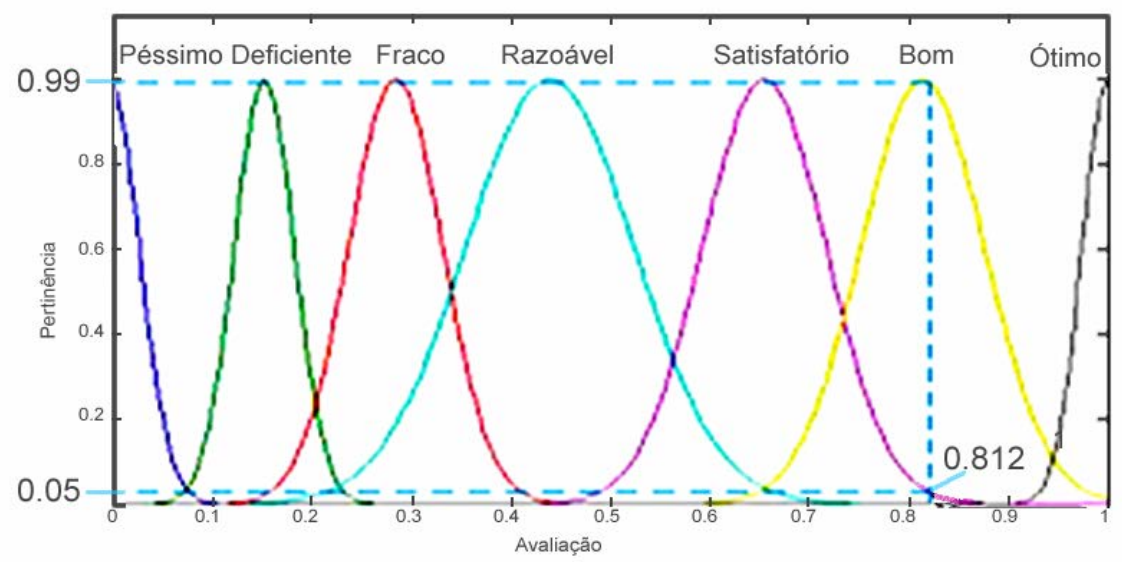

Figura 03: Pertinência a diferentes classes de eficiência para a DMU 122.

exemplos de avaliações calculadas nas duas simulações. O quadro apresenta o produtor (DMU), a avaliação com o método de análise envoltória de dados (DEA), a avaliação da Simulação 1 (Fuzzy Fatores) e Simulação 2 (Fuzzy Subjetivo).

A partir dos resultados das avaliações é possível notar que existem resultados diferentes entre a avaliação DEA e a fuzzy. Cabe destacar que o objetivo da proposta não é de obter exatamente os mesmo resultados da avaliação DEA, mas sim ter um método para tratar as incertezas dos parâmetros de entrada, como também classificar de forma gradual as avaliações, sem que, entretanto, exista diferença significativa com o método DEA.

Como expresso anteriormente, o arcabouço teórico sobre lógica fuzzy associa a cada elemento do conjunto um grau de pertinência. Como exemplo da demonstração dessa capacidade de classificação gradual inerente à lógica fuzzy é dado como exemplo o resultado do produtor de leite denominado DMU 122. Na Simulação 1 , na qual obteve-se resultado 1 no DEA, sendo classificado como "Ótimo" e com resultado 0,812 na avaliação fuzzy, resultando em aproximadamente $0,99 \%$ como "Bom" e 0,05\% como "Satisfatório". A Figura 03 apresenta graficamente o grau de pertinência em diferentes classes de eficiência.

\subsubsection{Módulo 4: Validação e teste de hipóteses}

Para confirmar se a proposta é valida foram efetuados dois testes estatísticos relacionando o resultado do DEA e da lógica fuzzy. A análise de variância permite afirmar a existência ou não de diferença significativa entre as médias das avaliações DEA e fuzzy. Para tanto, é necessário calcular o fator denominado (1) Anova: Fator Único e (2) Teste - Z: Duas amostras para médias para definir quanto à aceitação ou rejeição das hipóteses.

Portanto, os testes consistiram em verificar se existe diferença significativa entre os resultados da Análise Envoltória de Dados (DEA) e da Metodologia de Avaliação Fuzzy (MAF). Para isso, foram realizados dois testes estatísticos distintos com nível de significância de $5 \%$. Para os testes, a hipótese $\mathrm{H} 0$ permite afirmar que não existe diferença significativa entre as médias das avaliações. Como o objetivo desta etapa é validar a proposição, os testes estatísticos foram efetuados em todos os produtores avaliados e parcialmente apresentados no Quadro 08.

Os resultados das avaliações da Simulação 1 são apresentados na Tabela 01 e Tabela 02.

Os resultados da avaliação da Simulação 2 também são apresentados na Tabela 03 e Tabela04. 
Tabela 01: Cálculo da ANOVA entre o DEA x MAF utilizando excel - Simulação 1

\begin{tabular}{lllll}
\hline \hline \multicolumn{5}{l}{ ANOVA: Fator único dos DEA x MAF } \\
\hline Fonte da variação & Gl & $F$ & $p$ value & F crítico \\
Entre grupos & 1 & 1,8176 & 0,1784 & 3,8664 \\
\hline
\end{tabular}

Tabela 03 Cálculo da ANOVA entre o DEA x MAF utilizando excel - Simulação 2

\begin{tabular}{lcllll}
\hline \hline \multicolumn{4}{l}{ ANOVA: Fator único dos DEA x MAF } \\
\hline Fonte da variação & GI & $F$ & $p$ value & F crítico \\
Entre grupos & 1 & 0,0764 & 0,7823 & 3,8664 \\
\hline \hline
\end{tabular}

Obteve-se como resultado dos testes Anova e Teste $\mathrm{Z}$ a aceitação da hipótese $\mathrm{H} 0$. Ao aceitar a hipótese nula $\mathrm{H} 0$, confirma-se que não há diferença significativa entre as médias. Dessa forma, a validação do MAF proposto se apresenta como uma proposta viável de avaliação dos produtores de leite baseada na lógica fuzzy.

A seguir discutem-se alguns resultados da aplicação da Metodologia de Avaliação Fuzzy (MAF).

A partir do resultado da análise fatorial, pode-se destacar que identificaram-se quatro fatores/dimensões com a redução de metade das variáveis originais. Estas representam aproximadamente $94 \%$ da variabilidade dos dados, o que indica a alta representatividade dessas variáveis no universo de pesquisa.

Outro elemento observado é a constatação de que na análise envoltória de dados, o resultado da eficiência composta normalizada (que relaciona a eficiência padrão e eficiência invertida) é mais restritiva na definição do produtor como eficiente. Tomando como exemplo um cenário de simulação, enquanto o resultado da eficiência padrão define três produtores como eficientes, no índice de eficiência composta normalizada apenas um produtor
Tabela 02: Teste Z para o DEA x MAF utilizando excel - Simulação 1

\section{Teste Z para o DEA x MAF}

\begin{tabular}{ll}
\hline \hline Hipótese da diferença de média & 0 \\
\hline$P(Z<=z)$ uni-caudal & 0,0883
\end{tabular}

Tabela 04: Teste Z para o DEA x MAF utilizando excel - Simulação 2

\section{Teste Z para o DEA x MAF}

\begin{tabular}{ll}
\hline \hline Hipótese da diferença de média & 0 \\
\hline$P(Z<=z)$ uni-caudal & 0,1805 \\
\hline \hline
\end{tabular}

de leite é classificado como eficiente.

Ao analisar os resultados da análise de conglomerados, observa-se que é possível definir limiares entre as classes de eficiência e, dessa forma, relacionar os valores quantitativos e qualitativos das variáveis. Com isso, visualiza-se, por exemplo, que a característica de um produtor classificado como "ótimo" é possuir "ótimo" custo operacional total, custo unitário de produção e produtividade do capital, com "razoável" produtividade do custo operacional e "fraco" produtividade das vacas. Essa relação entre quantitativo/qualitativo permite maior entendimento das características da produção de leite.

Como pressuposto da lógica fuzzy, as variáveis linguísticas [Péssimo | Deficiente | Fraco | Razoável | Satisfatório | Bom | Ótimo] pertencem ao conjunto fuzzy [0 - 1] com grau de pertinência para cada elemento. Essa característica é observada nos resultados da avaliação proposta, apresentado no Quadro 09. Nota-se que a classificação baseada na lógica fuzzy permite identificar que um produtor de leite é classificado com graus de pertinência em mais de uma classe.

Quadro 09: Comparativo da avaliação DEA X Fuzzy.

\begin{tabular}{|l|l|l|l|l|}
\hline DMU & DEA & $\begin{array}{l}\text { Classe } \\
\text { Eficiência }\end{array}$ & $\begin{array}{l}\text { Simulação 2 } \\
\text { FUZzY Subjetivo }\end{array}$ & Classe FUZzY \\
\hline 122 & 1,000 & Ótimo & 0,967 & Bom 0.05 | Ótimo 0.40 \\
\hline 99 & 0,990 & Ótimo & 0,835 & Satisfatório 0.05 | Bom 0.85 \\
\hline 132 & 0,981 & Ótimo & 0,793 & Satisfatório 0.15 | Bom 0.83 \\
\hline 221 & 0,796 & Bom & 0,741 & Satisfatório 0.55 | Bom 0.35 \\
\hline 145 & 0,715 & Bom & 0,645 & Razoável 0.1 | Satisfatório 0.97 \\
\hline
\end{tabular}


Quadro 10: Comparativo de problemas DEA X Fuzzy.

\begin{tabular}{|l|l|l|}
\hline Problema & Análise Envoltória de Dados & Lógica Fuzzy \\
\hline $\begin{array}{lrl}\text { Outliers } \\
\text { observações não } \\
\text { usuais }\end{array}$ & $\begin{array}{l}\text { Resulta em avaliação incorreta da } \\
\text { eficiência }\end{array}$ & $\begin{array}{l}\text { O conhecimento de especialistas } \\
\text { na área de avaliação é utilizada } \\
\text { para construção das regras de } \\
\text { inferência }\end{array}$ \\
\hline Ruídos, tais como erro & Influência fortemente a avaliação \\
de medição & da eficiência & $\begin{array}{l}\text { inerente a lógica fuzzy o } \\
\text { tratamento da incerteza }\end{array}$ \\
\hline
\end{tabular}

Vilela et al. (2007) destacam alguns problemas que podem ocorrem com a utilização do método DEA. O Quadro 10 a seguir apresenta dois problemas que impactam o DEA e que não interferem na lógica fuzzy.

Com aceitação do modelo proposto, a principal vantagem da metodologia baseada na fuzzy é voltada ao produtor de leite. O sistema de inferência é construído com linguagem natural, não utiliza equações e melhora a tolerância para os dados subjetivos imprecisos. Estas características são importantes para melhor entendimento da produção pelos produtores de leite pois quase sempre não possuem uma base matemática sólida. Paes Souza et al. (2005) caracterizam o ambiente de gestão dos produtores de leite de Rondônia como sendo composto basicamente, por empresas familiares e administrados por produtores com baixa escolaridade.

\section{Conclusões}

A importância dos diversos grupos de agricultores familiares está relacionada ao considerável contingente de produtores na região Norte. Nesse sentido, faz-se necessário que se discutam formas de auxiliar o processo de tomada de decisão, uma das motivações do presente estudo.

Dessa forma, a abordagem fuzzy apresenta vantagens em relação ao método DEA: (1) naturalidade de sua abordagem a torna conceitualmente fácil de entender; (2) tolerância com dados imprecisos; (2) possibilidade de modelar funções não-lineares; (3) pode ser construída com base no conhecimento da área e; (4) baseada em linguagem natural, base da comunicação humana.

A estrutura sistematizada sustenta-se na objetividade de métodos multivariados para identificação, calculo e validação do modelo de avaliação de desempenho. De forma complementar, a lógica fuzzy proporciona que características como subjetividade, incerteza e imprecisão sejam tratadas na avaliação. Diante dos elementos expostos ao longo da investigação, a metodologia proposta se mostrou como uma alternativa viável, confirmado, inclusive, pelos testes estatísticos e, dessa forma, como alternativa para avaliação de desempenho dos produtores de leite.

\section{Referências}

ANTUNES, J.F.G; ZULLOJÚNIOR, J. Aplicação de lógica fuzzy para estimativa de área plantada da cultura de soja utilizando imagens AVHRR-NOAA. XIII Simpósio Brasileiro de Sensoriamento Remoto, Florianópolis, Brasil, 21-26 abril 2007, INPE, p. 3542.

ARAÚJO, A.G.; SARAIVA, A.M.; ANDRADE, M.T.C.A.; GUIMARÃES, M.F. Modelo Fuzzy para classificação da compactação do solo pelo tráfego de máquinas agrícolas em sistema conservacionista. V Congresso Brasileiro de Agroinformática, SBIAGRO. Londrina, 28 a 30 de setembro de 2005.

ARTES, A. Aspectos estatísticos da análise fatorial de escalas de avaliação. Revista de Psiquiatria Clínica. Volume 25. Número 5. Setembro / outubro de 1998. Disponível em <http://www.hcnet.usp.br/ipq/ revista/vol25/n5/index255.htm>. Acessado em 21 jul. 2011.

ASSIS SILVA, S.; SOUZA LIMA, J.S. Lógica fuzzy no mapeamento de variáveis indicadoras de fertilidade do solo. IDESIA (Chile) Volume 27, № 3, Septiembre-Diciembre, 2009.

BOGAN, C. E.; ENGLISH, M. J. Benchmarking: aplicações práticas e melhoria contínua. São Paulo: Makron Books, 1996.

CAMPOS FILHO, P. Método para apoio à decisão na verificação da sustentabilidade de uma unidade de conservação, usando a Lógica Fuzzy. 2004. 210f. Tese (Doutorado em Engenharia de Produção) - Curso de Pós-Graduação em Engenharia de Produção, Universidade Federal de Santa Catarina, 
Florianópolis, 2004.

CARVALHO, G. R. A Indústria de laticínios no Brasil: passado, presente e futuro. CEPEA - Centro de Estudos Avançados em Economia Aplicada ESALQ/USP. Boletim do Leite. 2010.

CHARNES, A.; COOPER, W. W.; RHODES, E. Measuring the efficiency of decision-making units. European Journal of Operational Research, p429444. 1978.

CORRAR, L. J.; DIAS FILHO, J. M. Análise multivariada: para os cursos de administração, ciências contábeis e economia. 1 ed. - 3. reimpr. São Paulo: Editora Atlas, 2001.

DIA, A.H.; MATHIAS, I.M.; BIAGGIONI, M.A.M.; CRUZ, L.F.; BILOBROVEC, M. Teoria Fuzzy aplicada à automação da condução racional da aeração de grãos de milho. V Congresso Brasileiro de Agroinformática, SBI-AGRO. Londrina, 28 a 30 de setembro de 2005.

EMBRAPA GADO DE LEITE - EGL. Disponível em: http://www.cnpgl.embrapa.br/. Acesso em: 26 jun. 2011.

FIGUEIREDO, MAD; MACEDO-SOARES, TDLVA; FUKS, S. Definição de atributos desejáveis para auxiliar a auto-avaliação dos novos sistemas de medição de desempenho organizacional. GESTÃO \& PRODUÇÃO, v.12, n.2, p.305-315, mai.-ago. 2005.

FUNDAÇÃO GETULIO VARGAS - FGV. Quem produz o que no campo: quanto e onde II: censo agropecuário 2006: resultados: Brasil e regiões / Fundação Getúlio Vargas, Instituto Brasileiro de Economia. Brasília: Confederação da Agricultura e Pecuária do Brasil, 2010. 192p.

FONTES, O.H.P.M.; MELLO, J. C. C. B. S. Aplicação da análise envoltória de dados na avaliação da qualidade do processo de fabricação de mananciais de linhas de eixo. V.04 n. 07. 2004.

GABRIEL FILHO, L. R. A. Application of fuzzy logic for the evaluation of livestock slaughtering. Eng. Agríc., Jaboticabal, v. 31, n. 4, 2011 . Available from <http://www.scielo.br/scielo.php? script=sci_ arttext\&pid=S0100-69162011000400019\&lng=en\&nr m=iso >. Acesso em: 26 abr. 2012.

GOMES, E.G.; MANGABEIRA, J.A.C. Uso de análise de envoltória de dados em agricultura: o caso de Holambra. ENGEVISTA, v. 6, n. 1, p. 19-27, abr. 2004.
MATARAZZO, D. C. Análise financeira de balanços: abordagem básica e gerencial. 3 ed. São Paulo: Atlas, 1995.

MEDEIROS, S., MELLO, R., FILHO CAMPOS, P. Análise de projetos para unidades de conservação, usando lógica fuzzy. Produção, v. 17, n. 2, p. 317329, Maio/Ago. 2007

MOORI, R. G.; ZILBER, M. A. Um estudo da cadeia de valores com a utilização da análise fatorial. Revista de administração contemporânea. Curitiba, v. 7, n. 3, Sept. 2003. Disponível em $<$ http://www. scielo.br/scielo.php?script=sci_arttext\&pid=S1415$65552003000300007 \& \operatorname{lng}=e n \& n r m=i s o>$. Acesso em: 26 abr. 2012.

NEELY, A.; GREGORY, M.; PLATTS, K. Performance measurement system design: A literature review and research agenda. International Journal of Production Economics. no. 4, pp 80-116; 1995.

PAES SOUZA, M.; SOUZAFILHO; ALVES, T.; CARNEIRO, G. Estratégias de Gestão da Agroindústria do Leite para Governança da Cadeia Produtiva em Rondônia. VIII Simpósio de Administração da Produção, Logística e Operações Internacionais - SIMPOI 2005 - FGV-EAESP.

PAES SOUZA, M.; SOUZAFILHO, T.A.; SERRA, N.E.M.; BORIS, M.; RIVA, F.R. A gestão da cadeia produtiva agroindustrial do leite e o mapeamento do arranjo produtivo local: Contribuição para o desenvolvimento de Rondônia. Sociedade Brasileira de Economia e Sociologia Rural. Fortaleza, 23 a 27 de Julho de 2006.

PEDRA, D.F.B.M.; GESSUIR, P.; SANTINI, G.A. Análise de fatores produtivos e comerciais da cadeia láctea no Brasil. Rio Branco - Acre, 20 a 23 de julho de 2008.

PEÑA, C. R. Um modelo de avaliação de eficiência da administração pública através do método análise envoltória de dados (DEA). Revista de Administração Contemporânea. n. 12, v.1, 2008, pp. 83-106.

QUEIRÓS, M.P.G.; NÄÄS, I.A. Estimativa de padrão de conforto ambiental para creche de suínos usando lógica fuzzy. V Congresso Brasileiro de Agroinformática, SBI-AGRO.Londrina, 28 a 30 de setembro de 2005.

RAMOS, J.O. Análise de eficiência de empresas de prestação de serviço pela DEA associada à análise fatorial / Joseli de Oliveira Ramos. - 2010. 
Dissertação (Mestrado) - Centro Universitário UNA, 2010. Programa de Mestrado em Turismo e Meio Ambiente.

REZENDE, M.L.;FERNANDES,L.P.D.;SILVA,A.M.R.. Utilização da análise fatorial para determinar o potencial de crescimento econonômico em uma região do sudeste do Brasil. Revista Economia e Desenvolvimento, n. 19, 2007.

RODRIGUES,M.H.S.. Avaliação de eficiência de produtores de leite utilizando análise envoltória de dados: o caso do município de Rolim de Moura no estado de Rondônia. Porto Velho, 2010. Dissertação (Mestrado).

RODRIGUES, F. Z.; SANTOS, S. A. A lógica fuzzy na administração de empresas. In: VII SEMEAD, SEMINÁRIOS DE ADMINISTRAÇÃO FEA/USP, de 10 a 11 de agosto de 2004. Disponível em: <http:// www.ead.fea.usp.br/semead/7semead/paginas/ artigos\%20recebidos/Adm\%20Geral/ADM25__A_L\%F3gica_Fuzzy_na_Administra\%E7\%E3o. PDF>. Acesso em: 28 abr 2012.

SANDRI, S.; CORREA, C. (1999) - Lógica Nebulosa. V Escola de Redes Neurais, Conselho Nacional de Redes Neurais. pp. c073-c090, 19 de julho. ITA, São José dos Campos - SP.

SHAW, I. S.; SIMÕES, M. G. (2007). Controle e Modelagem Fuzzy, 2 ed. Edgard Blücher Ltda, São Paulo, Brasil.

SINK, D.S; TUTTLE, T.C. Planejamento e Medição para a performance. Rio de Janeiro: Qualitymark, 1993.

STEFFANELLO, M; MACEDO, M. A. S.; ALYRIO, R. D. Eficiência produtiva de unidades agropecuárias: uma aplicação do método não-paramétrico análise envoltória de dados (DEA). Organizações Rurais \& Agroindustriais, Lavras, v.11, n.1, 2009.

VANTI, A; LINDSTAEDT, A; MIOTTO, G; PUGUES, L; MURARO, M. A controladoria utilizando a lógica fuzzy no auxílio à empresa para definição das prioridades do planejamento estratégico: um estudo em uma empresa de turismo. RAM Revista de Administração Mackenzie. Volume 8, n.1, 2007, p. 31-58.

VILELA, D.L.; NAGANO, M.S.; MERLO, E.M.. Aplicaçăo da Análise Envoltória de Dados em Cooperativas de Crédito Rural. RAC - Revista de Administração Contemporânea, 2a. Edição Especial, 2007, p. 99-120.
ZADEH, L. A.; Outline of a new approach to the Analysis of Complex Systems and Decision Processes, Journal IEEE Trans. On Systems, Man, And Cybernetics, Vol. SMC-3, №1, January 1973. ZADEH, L. A. (1965). Fuzzy Sets. Information and Control. pp. 338- 354. 